\title{
Iridium-Catalyzed Allylic Substitution of Silyl Dienolates
}

Metal-Catalyzed

Asymmetric

Synthesis and

Stereoselective

Reactions

\section{Key words}

allylic alkylation

dienolates

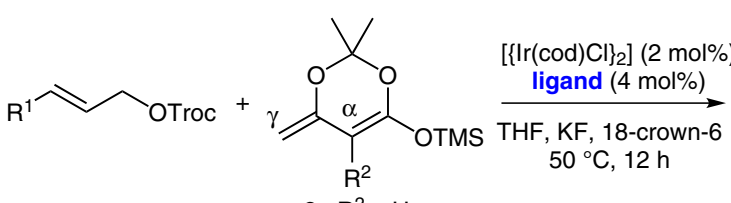

$$
\begin{aligned}
& \text { 2a } \mathrm{R}^{2}=\mathrm{H} \\
& \text { 2b } R^{2}=\mathrm{Me}
\end{aligned}
$$
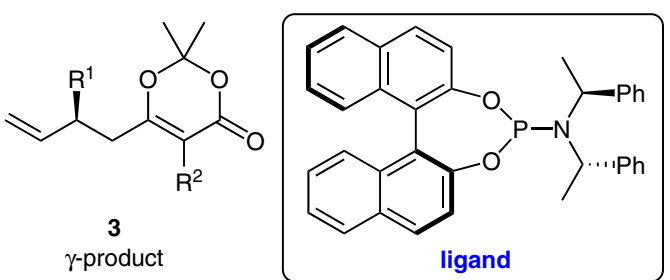

Selected examples of $2 \mathbf{a}$ (branched/linear $>20: 1)$ :<smiles>C=CC(CC1=CC(=O)OC(C)(C)O1)c1ccccc1</smiles>

$74 \%$ yield
$\alpha / \gamma=1: 10$ $\alpha / \gamma=1: 10$
$97 \%$ ee<smiles>C=CC(CC1=CC(=O)OC(C)(C)O1)c1ccccc1F</smiles>

$78 \%$ yield $\alpha / \gamma=1: 20$ $91 \%$ ee

Selected examples of $\mathbf{2 b}$ ( $\alpha$-products were not observed):<smiles>C=C[C@H](CC1=C(C)C(=O)OC(C)(C)O1)c1ccccc1</smiles>

$81 \%$ yield branched/linear $=15:$ $90 \%$ ee<smiles>C=C[C@@H](CC1=C(C)C(=O)OC(C)(C)O1)c1cccc(OC)c1</smiles>
branched/linear $=14: 1$ $92 \%$ ee<smiles>C=CC(CC1=CC(=O)OC(C)(C)O1)c1cn(C(=O)c2ccccc2)c2ccccc12</smiles>

$71 \%$ yield $\alpha / \gamma=1: 18$ $90 \%$ ee<smiles>C=C[C@H](CC1=C(C)C(=O)OC(C)(C)O1)c1cn(C(=O)OC(C)(C)C)c2ccccc12</smiles>

$84 \%$ yield branched/linear $>20: 1$ $90 \%$ ee<smiles>C=C[C@H](C)CC1=CC(=O)OC(C)(C)O1</smiles>

$63 \%$ yield $\alpha / \gamma=1: 9$ $90 \%$ ee

Derivatization of the product:<smiles>C=CC(CC(=O)CC(=O)OC)c1ccccc1</smiles>

$95 \%$ yield<smiles>C=C[C@H](CC1=CC(=O)OC(C)(C)O1)c1ccccc1</smiles>

$\mathrm{PhN}=\mathrm{C}=\mathrm{O}$<smiles>C=CC(Cc1cc(=O)n(-c2ccccc2)c(=O)o1)c1ccccc1</smiles>

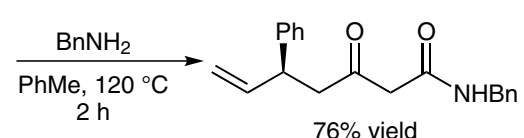

$76 \%$ yield
Significance: The allylic substitution at the $\gamma$-position of 1,3-dicarbonyl compounds has not been studied so far. Herein, the authors present the iridium phosphoramidite catalyzed allylic substitution reaction with high regio- and enantioselectivities. Silyl dienolates act as synthetic equivalents of $\beta$-keto ester dianions.
Comment: In this reaction, the combination of a leaving group on the allylic substrates and a chiral phosphoramidite ligand plays a crucial role to obtain high regioselectivities. The dioxinone moiety in the products can be converted easily into useful structures such as teramic acids. 